\title{
Stellar rotation as a new observable to test general relativity in the Galactic Center
}

\author{
Yun Fang ${ }^{1, *}$ and Xian Chen $^{2,1, \dagger}$ \\ ${ }^{1}$ Kavli Institute for Astronomy and Astrophysics at Peking University, Beijing 100871, China \\ ${ }^{2}$ Astronomy Department, School of Physics, Peking University, Beijing 100871, China
}

(Dated: December 22, 2020)

\begin{abstract}
S-stars in the Galactic Center are excellent testbeds of various general relativistic effects. While previous works focus on modeling their orbital motion around Sgr $\mathrm{A}^{*}$-the supermassive black hole in the Galactic Center-here we explore the possibility of using the rotation of S-stars to test the de Sitter precession predicted by general relativity. We show that by reorienting the rotation axes of S-stars, de Sitter precession will change the apparent width of the absorption lines in the stellar spectra. Our numerical simulations suggest that the newly discovered S4714 and S62 are best suited for such a test because of their small pericenter distances relative to Sgr A*. Depending on the initial inclination of the star, the line width would vary by as much as $20-76 \mathrm{~km} \mathrm{~s}^{-1}$ within a period of $20-40$ years. Such a variation is comparable to the current detection limit. Since the precession rate is sensitive to the orbital eccentricity and stellar quadrupole structure, monitoring the rotation of S-stars could also help us better constrain the orbital elements of the S-stars and their internal structures.
\end{abstract}

\section{INTRODUCTION}

The predictions of general relativity (GR) have been tested in a variety of astrophysical systems. They are tested with high precision in our solar system, e.g., the advance of the perihelion of Mercury ("Schwarzschild precession") [1], the "de Sitter" (or geodetic) precession in the earth-moon system due to the presence of the sun [2,3], and the Lense-Thrring precession of a man-made gyroscope orbiting around the earth [4]. GR also has passed test in the region of a much stronger field, such as in several pulsar systems [5,6], close to the supermassive black hole (SMBH) in M87 by imaging the shadow [7], as well as in the mergers of compact objects through detecting their gravitational waves [8-10].

The centers of galaxies are natural laboratories of strong gravity because they usually contain SMBHs [1113]. In fact, the Galactic Center also harbors a SMBH, known as Sagittarius $A^{*}\left(\mathrm{Sgr} \mathrm{A}^{*}\right)$, which has a mass of $4 \times 10^{6} M_{\odot}[14,15]$. It is surrounded by a dense cluster of young stars, called S-stars. Several of them, such as the famous S2 star, are orbiting Sgr A* with such a small distance and high eccentricity that the velocity reaches a few percent of the speed of light when the star passes the pericenter [16-19]. These stars are excellent testbeds of various relativistic effects. Using S2, we have detected the Schwarzschild precession [20] and the gravitational redshift of the spectral lines [21,22]. Continuous monitoring the orbits of S-stars would further allow us to test the "no hair theorem" [23, 24], constraining the possible fifth force of the unification theory [25], probing the hidden companion of Sgr $\mathrm{A}^{*}$ if there is one [26], and constraining the properties of the dark matter around the SMBH [27].

One essential component that is still missing in the current GR experiment using S-stars is stellar rotation. GR predicts that a rotating star, like a gyroscope, will undergo de Sitter precession around a SMBH. Just like the Schwarzschild precession, de Sitter precession is also a first-order post-Newtonian effect so that it becomes significant when the S-star passes the pericenter of its orbit around Sgr A*. It has been suggested that the de Sitter precession could be detected using a pulsar with an orbital period shorter than 100 years around Sgr A* [28], but so far no such pulsar has been discovered.

Two recent discoveries motivated us to study the de Sitter precession of S-stars. First, a few faint stellar objects were found on orbits closer than S2 relative to Sgr $A^{*}$ [19]. Their orbital eccentricities are also very large, making the pericenter distances much closer to Sgr A* as well. Second, the same observation revealed that at least one of the newly found S-stars, S4711, is fast rotating at a projected velocity of $V \sin i=239.60 \pm 25.21 \mathrm{~km} \mathrm{~s}^{-1}$. This projected rotation velocity is measured from the width of the absorption lines in the stellar spectrum. This result, as well as the earlier observation that S2 has a projected rotation velocity of $100 \pm 30 \mathrm{~km} \mathrm{~s}^{-1}$ [29], suggest that many, if not all, S-stars are fast rotators. Since a rotating star around $\mathrm{Sgr} \mathrm{A}^{*}$ should undergo de Sitter precession, we would expect the angle $i$ between the rotation axis and the line-of-sight to change whenever the star passes by the orbital perecenter. Such a change will induce a variation in the apparent line width, which could serve as a new observable for us to test GR in the Galactic Center.

\section{THEORY}

The precession law of a rotating star with a spin angular momentum $\mathbf{J}$ revolving around a $\mathrm{SMBH}$ is governed by the equation

$$
\frac{d \mathbf{J}}{d t}=\left(\boldsymbol{\Omega}^{(\text {geod })}+\boldsymbol{\Omega}^{(\mathrm{LT})}\right) \times \mathbf{J}+\mathbf{N}^{\text {quad }}
$$


(see, e.g. [30]), where $\boldsymbol{\Omega}^{\text {(geod) }}$ is the rate of geodetic precession determined by the mass $M$ of the SMBH, $\boldsymbol{\Omega}^{(\mathrm{LT})}$ is the Lense-Thirring precession rate determined by the spin angular momentum $\mathbf{S}$ of the $\mathrm{SMBH}$, and $\mathbf{N}^{\text {quad }}$ is the torque induced by the interaction between the mass quadrupole moment of the star and the tidal force of the SMBH.

To facilitate the calculation, we define $\mathbf{r}$ as the relative distance from the $\mathrm{SMBH}$ to the star, so that $\mathbf{v}=\dot{\mathbf{r}}$ is the orbital velocity of the star and $\mathbf{n}:=\mathbf{r} / r$ is the direction vector. With these terms, we can write

$$
\mathbf{\Omega}^{\text {(geod) }}=\frac{3}{2} \frac{M}{r^{2}} \mathbf{v} \times \mathbf{n} .
$$

We have assumed $G=c=1$ in the above calculation. The precession rate due to the Lense-Thirring effect can be calculated with

$$
\boldsymbol{\Omega}^{(\mathrm{LT})}=\frac{1}{r^{3}}[-\mathbf{S}+3 \mathbf{n}(\mathbf{n} \cdot \mathbf{S})],
$$

with is of order $\mathcal{O}(v)$ smaller than the geodetic precession and hence neglected in the later analysis.

The last term $\mathbf{N}^{\text {quad }}$ in Equation (1) is related to the stellar mass quadrupole moment tensor $\mathbf{Q}$ as

$$
\mathbf{N}^{\text {quad }}=-3 \frac{M}{r^{3}} \mathbf{n} \times \stackrel{\leftrightarrow}{\mathbf{Q}} \cdot \mathbf{n},
$$

and he components of $\mathbf{Q}$ is given by

$$
Q^{j k}=\left[\int \rho x^{j} x^{k} d^{3} x\right]^{\mathrm{STF}},
$$

where $\rho$ denotes the density of the star and the superscript "STF" means the symmetric and trace-free part.

To be self-consistent, we assume that the nonvanishing part of the mass quadrupole moment is induced by the rotation of the star. Under the symmetry condition and in the coordinate system $\left(x^{\prime}, y^{\prime}, z^{\prime}\right)$ where $z^{\prime}$ is aligned with the $\mathbf{J}$ axis, the tensor $\mathbf{Q}$ takes a simple form in which $I_{x^{\prime} y^{\prime}}=I_{x^{\prime} z^{\prime}}=I_{y^{\prime} z^{\prime}}=0$ and $I_{x^{\prime} x^{\prime}}=I_{y^{\prime} y^{\prime}} \neq I_{z^{\prime} z^{\prime}}$. It follows that

$$
Q^{i^{\prime} j^{\prime}}=\left(I_{x^{\prime} x^{\prime}}-I_{z^{\prime} z^{\prime}}\right)\left(\begin{array}{ccc}
\frac{1}{3} & 0 & 0 \\
0 & \frac{1}{3} & 0 \\
0 & 0 & -\frac{2}{3}
\end{array}\right) .
$$

To further derive the component of $\mathbf{Q}$ in an arbitrary coordinate system $(x, y, z)$, we use the transformation matrix

$$
R(i, \kappa)=\left(\begin{array}{ccc}
\cos \kappa & \sin \kappa & 0 \\
-\sin \kappa & \cos \kappa & 0 \\
0 & 0 & 1
\end{array}\right)\left(\begin{array}{ccc}
1 & 0 & 0 \\
0 & \cos i & \sin i \\
0 & -\sin i & \cos i
\end{array}\right),(7)
$$

where $i$ is the angle between the $z$ and $z^{\prime}$-axis, $\kappa$ is the angle between the $y$-axis and the projection of the $z^{\prime}$-axis in the $x-y$ plane, thus $\left(i, \frac{\pi}{2}-\kappa\right)$ is the spherical coordinate of the $z^{\prime}$-axis in the $(x, y, z)$ frame. Then we can derive $Q^{i j}$ in the $(x, y, z)$ frame using the transformation $Q=R Q^{\prime} R^{\mathrm{T}}$.

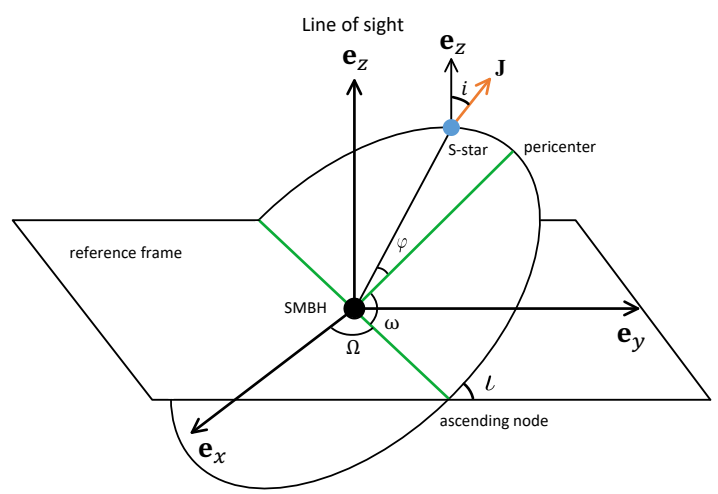

FIG. 1. Definition of the orbital elements of an S-star (blue dot) in a reference frame centered on the SMBH (black dot) and with the $z$-axis aligned with the line-of-sight (observer resides in the $-z$ direction). The orange arrow shows the rotating axis, i.e., the direction of the spin angular momentum, of the star.

\section{CONNECTING THEORY WITH OBSERVATION}

If the direction of $\mathbf{J}$ varies, the rotation velocity projected along the line-of-sight, $V \sin i$, will change accordingly. The projected velocity $V \sin i$ is related to the observed width $\Delta \lambda$ of the absorption lines in the spectra of S-stars. The relationship is $\Delta \lambda=2 \lambda V \sin i$, where $\lambda$ is the wavelength of the centroid of an spectral line. Therefore, we can use the variation of $\Delta \lambda$ to test the prediction of Equation (1).

In the following, we simulate the evolution of $V \sin i$ for four S-stars, namely S4711, S4714, S62, and S2, by numerically integrating Equation (1). S2 is the most famous one due to its early detection, closeness to Sgr A*, and large eccentricity. It has been monitored for almost three decades by SINFONI, NACO, and, more recently, GRAVITY [20, 21]. The orbital period is about 16 years and the eccentricity is about 0.88 . The three newly discovered S-stars, S4711, S4714 and S62, have even shorter periods than S2 as well as relatively large orbital eccentricities [19]. These properties make them ideal test subjects of the de Sitter precession. The orbital parameters of our chosen S-stars are given in Table I, and the meanings of the parameters are illustrated in Figure 1.

We set the mass of the SMBH to be $M=4 \times 10^{6} M_{\odot}$. We assume that the stellar rotation velocity in the equatorial plane is $V=300 \mathrm{~km} \mathrm{~s}^{-1}$, to be consistent with the detected velocity in [19]. We note that changing the initial $V$ will not affect the evolution of $i$. Therefore, if we adopt a lower rotation velocity, the projected line width and its variation with time would be proportionally smaller.

The quadrupole of the star is computed with

$$
I_{x^{\prime} x^{\prime}}=I_{y^{\prime} y^{\prime}}=(1+q) I_{z^{\prime} z^{\prime}}=\frac{\epsilon m_{*} r_{*}{ }^{2}}{\sqrt{2+1 /(1+q)^{2}}},
$$


TABLE I. The orbital parameters and simulation results for S2, S4711, S4714, and S62.

\begin{tabular}{cccccccccc}
\hline \hline name & $m_{*}\left(M_{\odot}\right)$ & $a(\mathrm{mpc})$ & $e$ & $\iota\left(^{\circ}\right)$ & $\omega\left(^{\circ}\right)$ & $\Omega\left(^{\circ}\right)$ & $t_{\text {period }}$ (years) & $\Delta V(q=0)$ & $\Delta V(q=0.1)$ \\
\hline $\mathrm{S} 2$ & 10 & 4.895 & 0.886 & 133.9 & 66.0 & 227.4 & 16 & $0.4 \mathrm{~km} \mathrm{~s}^{-1}$ & $0.6 \mathrm{~km} \mathrm{~s}^{-1}$ \\
$\mathrm{~S} 4711$ & 2.2 & 3.002 & 0.768 & 114.71 & 131.59 & 20.10 & 7.6 & $0.4 \mathrm{~km} \mathrm{~s}^{-1}$ & $0.4 \mathrm{~km} \mathrm{~s}^{-1}$ \\
$\mathrm{~S} 4714$ & 2.0 & 4.079 & $0.985 \pm 0.011$ & 127.70 & 357.25 & 129.28 & 12.0 & $53 \mathrm{~km} \mathrm{~s}^{-1}$ & $76 \mathrm{~km} \mathrm{~s}^{-1}$ \\
$\mathrm{~S} 62$ & 6.1 & 3.588 & $0.976 \pm 0.01$ & 72.76 & 42.62 & 122.61 & 9.9 & $21 \mathrm{~km} \mathrm{~s}^{-1}$ & $25 \mathrm{~km} \mathrm{~s}^{-1}$ \\
\hline
\end{tabular}

Notes: The orbital parameters of S2 are from [29] and [22], and those of S4711, S4714, and S62 are from [19]. For each star we adopt from the above references the mass $m_{*}$, semi-major axis $a$, inclination $\iota$, phase of pericenter $\omega$, angle of ascending node $\Omega$, and orbital period $t_{\text {period }}$. The last two columns $\Delta V(q=0)$ and $\Delta V(q=0.1)$ are the maximum variation of the line width, $V \sin i$, within a period of 40 years computed using two different stellar ellipticities, $q=0$ and $q=0.1$.

where $m_{*}$ is the mass of the star, $r_{*}$ is the stellar radius, $q$ is the ellipticity of the star, and $\epsilon$ is a parameter accounting for the internal mass distribution of the star. Moreover, we compute the spin angular momentum of the star with $\mathbf{J}=\epsilon_{J} m_{*} r_{*} V \mathbf{j}$, where $\mathbf{j}:=\mathbf{J} /|\mathbf{J}|$ is the direction of $\mathbf{J}$ and $\epsilon_{J}$ is another dimensionless parameter characterizing the internal rotating structure of the star.

We note that the result of the numerical integration is insensitive to the internal structure of the star but mainly depends on the size $r_{*}$ and the ellipticity $q$. The reasons are as follows. First, the stellar mass $m_{*}$ cancels out on both sides of Equation (1) because both $\mathbf{J}$ and $\mathbf{N}^{\text {quad }}$ are proportional to $m_{*}$. Second, the parameter $\epsilon$ in $\mathbf{N}^{\text {quad }}$ and the parameter $\epsilon_{J}$ in $\mathbf{J}$ also cancel out if the star rotates as a rigid body, because in this case both parameters scale with $\int \rho r^{\prime 4} d r^{\prime}$. For non-rigid-body rotation, $\epsilon$ and $\epsilon_{J}$ would be different, which would result in a different relative contribution to the precession rate by the two terms on the right-hand-side of Equation (1). However, numerically, the net effect can be absorbed into the uncertainty of the value of $q$.

We adopt a value of $r_{*}=10 R_{\odot}$ for S2 [29]. For the other three S-stars, we derive their radii from the massradius relationship presented in [31]. For the ellipticity $q$, since there is currently no observational constraint, we take 0 and 0.1 as two representative values. The latter value is predicted by the model of early-type stars [32].

\section{RESULTS}

S4714 has the smallest pericenter distance and hence is best suited for the test of the de Sitter precession. Figure 2 shows the evolution of the orientation of $\mathbf{J}$ within 40 years. We tried seven different initial conditions for the inclination angle, namely, $i_{0}=10^{\circ}, 30^{\circ}, 60^{\circ}, 90^{\circ}$, $120^{\circ}, 150^{\circ}$, and $170^{\circ}$. The right ordinate corresponds to the variation of the apparent (projected) rotation velocity, $|\Delta V|$, which is defined as $\left|V \sin i-V \sin i_{0}\right|$. We also allowed the orbital eccentricity $e$ to vary between the medium and maximum values allowed by observation, and the stellar ellipticity $q$ to vary between 0 and 0.1 , to accommodate the observational and theoretical uncertainties.

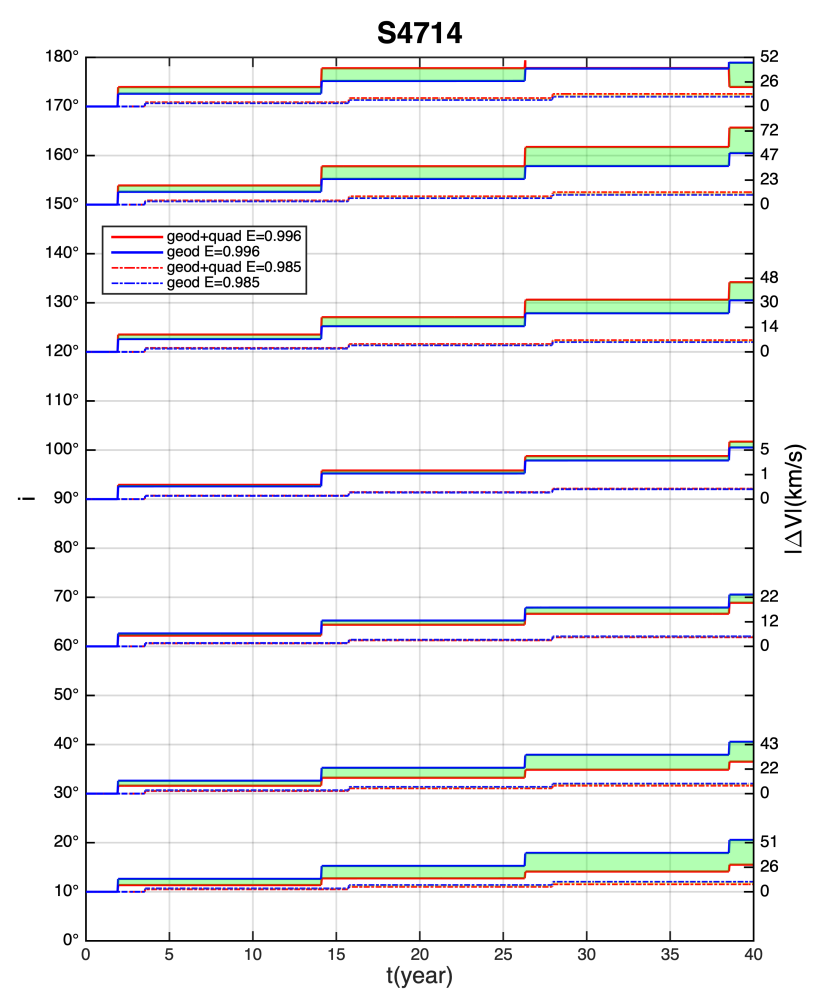

FIG. 2. Evolution of the inclination angle (left ordinate) for S4714, as well as the corresponding variation of the apparent (projected) rotation velocity (right ordinate). We tried seven different initial values for the inclination angle, while the initial azimuth angle of the rotation axis $(\mathbf{j})$ is fixed to $120^{\circ}$. The dot-dashed lines are the results derived using the medium value $e=0.985$ of the orbital eccentricity, and the solid lines are derived using the maximum eccentricity $e=0.996$ allowed by observation within the uncertainty. The red lines are computed assuming that the quadrupole momentum of the star is zero and the blue ones are assuming $q=0.1$. Therefore, the shaded area between the red and blue lines indicates the uncertainty due to the unknown stellar structure. The sudden jumps in the evolution of $i$ coincide with the star passing by the pericenter, where both the de Sitter precession and the mass-quadrupole effect become the most significant. 
Figure 2 shows that larger orbital eccentricity in general leads to a larger variation of the projected rotation velocity $|\Delta V|$. Moreover, the quadrupole moment of the star suppresses the precession of the stellar rotation axis when $i<90^{\circ}$ and enhances the precession when $i \geq 90^{\circ}$. These results indicate that measuring $|\Delta V|$ would enable us to better constrain the orbital elements as well as probe the internal structure of S4714.

Regarding detectability, if $e=0.985$, i.e., the medium value given by observation, we find that $|\Delta V|$ remains smaller than the current detection limit, $25 \mathrm{~km} \mathrm{~s}^{-1}$ [19], during the 40 years of evolution. However, when $e$ increases to the maximum value allowed by observation, i.e., $e=0.996,|\Delta V|$ could exceed $25 \mathrm{~km} \mathrm{~s}^{-1}$ within 20 years if the initial inclination angle is $i_{0}=10^{\circ}, 30^{\circ}, 150^{\circ}$, or $170^{\circ}$. If the observational period could be elongated to 40 years, an initial inclination angle of $i_{0}=120^{\circ}$ would also lead to an detectable $|\Delta V|$. Moreover, if in the future the detection limit of the rotation velocity could be improved to $20 \mathrm{~km} \mathrm{~s}^{-1}$, the variation $|\Delta V|$ induced by an initial inclination angle of $i_{0}=60^{\circ}$ would also become detectable within a period of 40 years. To detect the $|\Delta V|$ corresponding to an initial inclination of $i_{0}=90^{\circ}$, an observational period of 40 years and a detection limit of $5 \mathrm{~km} \mathrm{~s}^{-1}$ are preferred. For easier comparison with other stars, the maximum $|\Delta V|$ from our numerical simulations are given in the last two columns of Table I.

S62 also has a relatively small pericenter distance. The simulation results for this star are shown in Figure 3. Comparing with the previous results for S4714, we find two differences. First, the variation of the projected rotation velocity is much smaller than that of S4714. Second, the lines taking into account the quadrupole moment of the star (red) are less separated from the line without the quadrupole moment (blue). Both differences are caused by the larger pericenter distance of $\mathrm{S} 62$ relative to that of S4714. Because of the larger pericenter distance, the magnitude of $\boldsymbol{\Omega}^{\mathrm{geod}}$, which determines the rate of de Sitter precession, and the ratio $\left|\mathbf{N}^{\text {quad }}\right| /\left|\mathbf{\Omega}^{\text {geod }} \times \mathbf{J}\right|$, which determines the relative importance of the quadrupole moment, both become smaller. Nevertheless, when we set $e=0.986$ (solid lines), i.e., the maximum eccentricity allowed by observation, the variation of $V \sin i$ within 40 years is comparable to the current detection limit of $25 \mathrm{~km} \mathrm{~s}^{-1}$ if $i_{0}=10^{\circ}$ or $170^{\circ}$. The maximum values of $|\Delta V|$ are also given in Table $\mathrm{I}$.

S2 and S4711 have larger pericenter distances than those of $\mathrm{S} 4714$ and $\mathrm{S} 16$. For this reason, their $|\Delta V|$ is too small to be detected. We do not show the evolution of their inclination angles but only give the maximum $|\Delta V|$ derived from our numerical simulations in Table I.

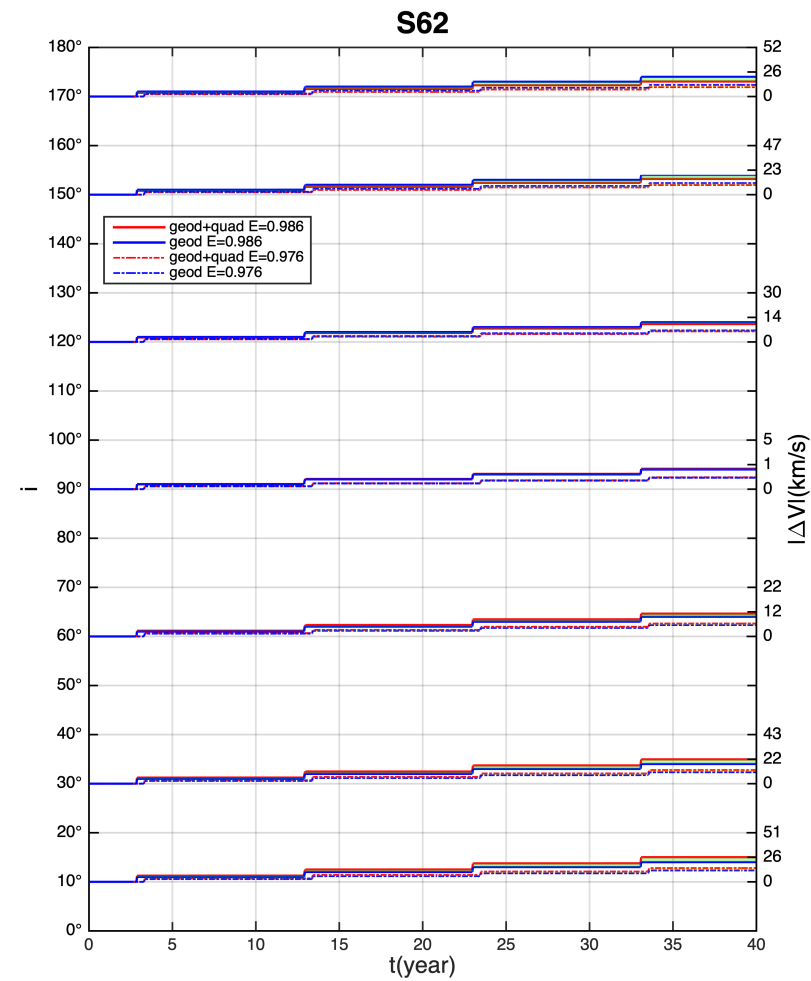

FIG. 3. The same as Fig. 2 but for S62.

\section{CONCLUSION}

In this Letter, we pointed out that the de Sitter precession induced by Sgr $\mathrm{A}^{*}$ on the surrounding S-stars could be detected through the variation of the width of the spectral lines. Our numerical simulations suggested that the newly discovered S4717 and S62 are best suited for such a test because of their small pericenter distances relative to $\operatorname{Sgr} \mathrm{A}^{*}$. We showed that within a period of $20-40$ years, their line width would vary by as much as $20-76, \mathrm{~km} \mathrm{~s}^{-1}$, depending on the initial inclination of the rotation axes. Such a variation is comparable to the current detection limit. Since the precession rate is sensitive to the eccentricity of the stellar orbit as well as the quadrupole moment of the star, observing the variation of the line width would also help us better constrain the orbital elements and probe the internal structures of the S-stars.

This work is supported by the National Science Foundation of China (NSFC) grant No 11721303. XC acknowledges the support by NSFC grants No 11873022, and 11991053. 
* fang.yun@pku.edu.cn

$\dagger$ xian.chen@pku.edu.cn

[1] C. M. Will, Theory and Experiment in Gravitational Physics (Cambridge University Press, 1993).

[2] I. I. Shapiro, R. D. Reasenberg, J. F. Chandler, and R. W. Babcock, Phys. Rev. Lett. 61, 2643 (1988).

[3] J. G. Williams, D. H. Boggs, J. O. Dickey, and W. M. Folkner, .

[4] I. Ciufolini and E. C. Pavlis, Nature 431, 958 (2004).

[5] M. Kramer, I. H. Stairs, R. N. Manchester, M. A. McLaughlin, A. G. Lyne, R. D. Ferdman, M. Burgay, D. R. Lorimer, A. Possenti, N. D'Amico, J. M. Sarkissian, G. B. Hobbs, J. E. Reynolds, P. C. C. Freire, and F. Camilo, Science 314, 97 (2006), arXiv:astroph/0609417 [astro-ph].

[6] M. Kramer, Int. J. Mod. Phys. D 25, 1630029 (2016), arXiv:1606.03843 [astro-ph.HE].

[7] K. Akiyama et al. (Event Horizon Telescope), Astrophys. J. 875, L1 (2019), arXiv:1906.11238 [astro-ph.GA].

[8] B. P. Abbott et al. (LIGO Scientific, Virgo), Phys. Rev. Lett. 116, 061102 (2016), arXiv:1602.03837 [gr-qc].

[9] B. P. Abbott et al. (LIGO Scientific, Virgo), Phys. Rev. X9, 031040 (2019), arXiv:1811.12907 [astro-ph.HE].

[10] R. Abbott et al., arXiv e-prints , arXiv:2010.14527 (2020), arXiv:2010.14527 [gr-qc].

[11] A. Ghez, M. Morris, J. Lu, N. Weinberg, K. Matthews, T. Alexander, P. Armitage, E. Becklin, W. Brown, R. Campbell, T. Do, A. Eckart, R. Genzel, A. Gould, B. Hansen, L. Ho, F. Lo, A. Loeb, F. Melia, D. Merritt, M. Milosavljevic, H. Perets, F. Rasio, M. Reid, S. Salim, R. Schödel, and S. Yelda, in astro2010: The Astronomy and Astrophysics Decadal Survey, Vol. 2010 (2009) p. 89, arXiv:0903.0383 [astro-ph.GA].

[12] J. Kormendy and L. C. Ho, Annual Review of Astronomy and Astrophysics 51, 511 (2013), https://doi.org/10.1146/annurev-astro-082708-101811.

[13] N. J. McConnell and C.-P. Ma, Astrophys. J. 764, 184 (2013), arXiv:1211.2816 [astro-ph.CO].

[14] A. M. Ghez, S. Salim, N. N. Weinberg, J. R. Lu, T. Do, J. K. Dunn, K. Matthews, M. R. Morris, S. Yelda, E. E. Becklin, T. Kremenek, M. Milosavljevic, and J. Naiman, Astrophys. J. 689, 1044 (2008), arXiv:0808.2870 [astroph].

[15] R. Genzel, F. Eisenhauer, and S. Gillessen, Rev. Mod. Phys. 82, 3121 (2010).

[16] R. Schödel, T. Ott, R. Genzel, R. Hofmann, M. Lehnert, A. Eckart, N. Mouawad, T. Alexander, M. J. Reid, R. Lenzen, M. Hartung, F. Lacombe, D. Rouan, E. Gendron, G. Rousset, A. M. Lagrange, W. Brandner,
N. Ageorges, C. Lidman, A. F. M. Moorwood, J. Spyromilio, N. Hubin, and K. M. Menten, Nature 419, 694 (2002), arXiv:astro-ph/0210426 [astro-ph].

[17] L. Meyer, A. M. Ghez, R. Schödel, S. Yelda, A. Boehle, J. R. Lu, T. Do, M. R. Morris, E. E. Becklin, and K. Matthews, Science 338, 84 (2012), arXiv:1210.1294 [astro-ph.GA].

[18] A. Boehle, A. M. Ghez, R. Schödel, L. Meyer, S. Yelda, S. Albers, G. D. Martinez, E. E. Becklin, T. Do, J. R. Lu, K. Matthews, M. R. Morris, B. Sitarski, and G. Witzel, The Astrophysical Journal 830, 17 (2016).

[19] F. Peißker, A. Eckart, M. Zajaček, B. Ali, and M. Parsa, Astrophys. J. 899, 50 (2020), arXiv:2008.04764 [astroph.GA].

[20] R. Abuter et al. (GRAVITY), Astron. Astrophys. 636, L5 (2020), arXiv:2004.07187 [astro-ph.GA].

[21] R. Abuter et al. (GRAVITY), Astron. Astrophys. 615, L15 (2018), arXiv:1807.09409 [astro-ph.GA].

[22] T. Do, A. Hees, A. Ghez, G. D. Martinez, D. S. Chu, S. Jia, S. Sakai, J. R. Lu, A. K. Gautam, K. K. O'Neil, E. E. Becklin, M. R. Morris, K. Matthews, S. Nishiyama, R. Campbell, S. Chappell, Z. Chen, A. Ciurlo, A. Dehghanfar, E. Gallego-Cano, W. E. Kerzendorf, J. E. Lyke, S. Naoz, H. Saida, R. Schödel, M. Takahashi, Y. Takamori, G. Witzel, and P. Wizinowich, Science 365, 664 (2019), arXiv:1907.10731.

[23] C. M. Will, The Astrophysical Journal 674, L25 (2008).

[24] D. Merritt, T. Alexander, S. Mikkola, and C. M. Will, Phys. Rev. D 81, 062002 (2010), arXiv:0911.4718 [astroph.GA].

[25] A. Hees et al., Phys. Rev. Lett. 118, 211101 (2017), arXiv:1705.07902 [astro-ph.GA].

[26] S. Naoz, C. M. Will, E. Ramirez-Ruiz, A. Hees, A. M. Ghez, and T. Do, The Astrophysical Journal 888, L8 (2019).

[27] E. Becerra-Vergara, C. Arguelles, A. Krut, J. Rueda, and R. Ruffini, Astron. Astrophys. 641, A34 (2020), arXiv:2007.11478 [astro-ph.GA].

[28] E. Pfahl and A. Loeb, The Astrophysical Journal 615, 253 (2004).

[29] F. Martins, S. Gillessen, F. Eisenhauer, R. Genzel, T. Ott, and S. Trippe, Astrophys. J. Lett. 672, L119 (2008), arXiv:0711.3344 [astro-ph].

[30] K. S. Thorne and J. B. Hartle, Phys. Rev. D31, 1815 (1984).

[31] C. A. Tout, O. R. Pols, P. P. Eggleton, and Z. Han, Monthly Notices of the Royal Astronomical Society 281, 257 (1996), https://academic.oup.com/mnras/articlepdf $/ 281 / 1 / 257 / 18540111 / 281-1-257 . p d f$.

[32] D. Gagnier, M. Rieutord, C. Charbonnel, B. Putigny, and F. Espinosa Lara, Astronomy\&Astrophysics 625, A89 (2019), arXiv:1904.05219 [astro-ph.SR]. 